\title{
The Governance Mechanism of Knowledge Sharing Hostility within E-business Enterprise
}

\author{
Liang Zhao ${ }^{1}$, Shuhui Fan ${ }^{1}$ and Ruihua Wang ${ }^{1}$ \\ ${ }^{1}$ Management Engineering Department, Zhengzhou University, Zhengzhou, China \\ Corresponding Email: fsh0209@163.com
}

\begin{abstract}
Organizational knowledge sharing is the key to improve the core competitiveness and production efficiency. How to govern the behaviour of knowledge sharing and promote knowledge sharing has become the focus of organizational knowledge governance. Under the exploration of the relationship between knowledge governance, knowledge sharing hostility and knowledge sharing within the Internet organization and empirical analysis, we draw the following conclusions: formal and informal knowledge governance have a negative and weak impact on the reasons of knowledge hoarding and rejecting and the attitude toward the failure knowledge; The reasons of knowledge hoarding and rejecting have a significant and negative impact on knowledge sharing, but passive attitudes towards failure knowledge have a positive influence on knowledge sharing; The reasons of knowledge rejecting and passive attitudes towards failure knowledge exist partial mediating effect between formal and informal knowledge governance and knowledge sharing, but the reasons of the knowledge hoarding does not exist.
\end{abstract}

Keywords. Knowledge governance, knowledge sharing hostility, knowledge sharing, mediating effect.

\section{Introduction}

Organizations can integrate internal and external knowledge by knowledge sharing within the organization, which can help enterprises to find the leakage of the original knowledge system, help companies to get out of the governance blind area, form a new knowledge system and produce more innovative ideas [1]. Knowledge, as a kind of intangible assets, has two attributes of "individual property" and "public goods". In the process of knowledge sharing, there are two opposing rights: proprietary right of knowledge and knowledge sharing [2]. It is the mutual restriction of these two kinds of rights that make the social dilemma when knowledge sharing is carried out. From the perspective of enterprise, knowledge sharing is a kind of collective behaviour, and employees should select organization's common interests instead of personal interests [3]. Similarly, some people contend that knowledge is intangible private property. When the organization takes the heterogeneous knowledge as an important indicator to evaluate the value of staffs, the staffs will repel knowledge sharing in order to preserve their own competitive advantage, characterized by knowledge hoarding, rejection behaviour and attitude towards the errors of knowledge transformation, etc. Snejina (2003) called this kind of behaviour of not willing to share knowledge as knowledge sharing hostility. Studies show that the behaviour of knowledge sharing hostility is common in the organization. Enterprises will take the corresponding knowledge governance mechanism to break the phenomenon of knowledge sharing hostility in order to enhance the overall core competitive advantage.

E-commerce is a kind of business activity that using information technology to optimize of enterprise business activities continuously, and e-commerce enterprises are those organizations who engaged in e-commerce activities. Ecommerce enterprises are high-tech industries and knowledge intensive enterprises, whose operations cannot be separated from the knowledge sharing between employees. Luo (2010) pointed out that the core elements of the hightech industry are the staff education, experience and professional quality and so on, exclusively and fuzzily [4]. It is these characteristics that cause the enterprise's dilemma of knowledge sharing. In previous researches on knowledge sharing, few domestic scholars have studied the hostility of knowledge sharing, while the research on the governance mechanism of knowledge sharing hostility in the e-commerce enterprise is more scarce. This paper studies the governance mechanism of knowledge sharing hostility in the e-commerce enterprise based on the social dilemma theory and the theory of knowledge governance mechanism (KGM). 


\section{Social dilemma theory}

\subsection{Social dilemma}

In the knowledge-intensive enterprises, the heterogeneous knowledge is an important source of core competitiveness of enterprises, and the heterogeneous knowledge grasped by employees is a manifestation of their competitive advantage. Heng-Li Yang (2008) found that employees are not willing to share their knowledge in order to maintain their position in the organization, but the organization is bound to taking certain measures to promote knowledge sharing from the perspective of overall interest, which causes governance dilemma between the individual and organizational, namely "social dilemma" [5]. Wang (2015) pointed out that the main problem of knowledge governance lies in the knowledge sharing hostility among employees [6]. There are three main aspects of knowledge sharing behaviour: knowledge hoarding, knowledge rejecting and negative attitude towards mistakes. Knowledge Hoarding refers to the behaviour of employees unwilling to share their knowledge with others; knowledge rejecting refers to the behaviour of employees unwilling to accept others' knowledge in knowledge sharing; false knowledge refers to the mistakes staff made in the work or what they have learned from the failure knowledge, and their attitude towards mistakes will be different because of different management system within the organization and personal different subjective feelings. Diverse personal interest of employees, differentiation of educational background and knowledge structure, different jobs will affect knowledge sharing behaviour the organization. The greater the knowledge distance is between employees, the more difficult they acquire knowledge from each other, the less likely it is to occur knowledge sharing behaviour [7].

\subsubsection{Reasons of knowledge hoarding and knowledge sharing}

There are two main reasons for the knowledge hoarding: individual reasons and the organizational environment. From personal point of view, employees are afraid of losing their competitive advantages after knowledge sharing, who want to retain their absolute control right of knowledge and reserve their own personal competitive advantage [8]; in addition, the staff think they can attain nothing in knowledge sharing. If they spend their time and energy on their work rather than on knowledge sharing, their capacity will be further improved and more conducive to their promotion [8]. In terms of organizational environment, when sharing knowledge, employees are in a hostile environment, which means that colleagues regard their knowledge with suspicion. If so, knowledge sharing and cannot achieve the desired results and will produce exactly the opposite result [8]. Therefore, employees do not want to share their knowledge in such an environment.

Reasons of knowledge hoarding in e-business enterprise: One is related to the organizational atmosphere. Unfriendly and unfamiliar colleagues tend to choose knowledge hoarding because they don't have enough trust [9]. Second, they worry that their knowledge is false. They will mislead colleagues if sharing false knowledge.

Thus, we propose:

H1: Reasons of knowledge hoarding have a negative effect on knowledge sharing.

\subsubsection{Reasons of knowledge rejecting and knowledge sharing}

Reasons of knowledge rejecting also has two aspects: individual and organization. From a personal point of view, first, knowledge receivers may not trust the quality of others' knowledge and they have a fear that external knowledge will bring them trouble. Another reason may be personal and professional pride means that some argue that they have grasped enough knowledge so there is no need to accept others' knowledge. From the organizational standpoint, corporations refuse to learn from competitors, lack of trust in other enterprise and lack of a sense of belonging for external knowledge [10].

In addition to the reasons mentioned above, another important reason of knowledge rejecting in e-business enterprise is that demanding of knowledge areas is different. The knowledge provided by the other party is not their own needs, then knowledge sharing the two sides will repel each other the knowledge passed. If the knowledge that the opposite party offers is not what they want, both of the knowledge receiver and knowledge provider will reject knowledge sharing.

Therefore, we hypothesize that:

$\mathrm{H} 2$ : Reasons of knowledge rejecting have a negative effect on knowledge sharing.

\subsubsection{Passive attitudes toward false knowledge and knowledge sharing}

When it comes to mistakes, most people always hide them instead of sharing. Their own psychological perception is one of the reasons. They are afraid of that others look at them strangely. Another explanation is that they worry their leaders blame them or deduct wages and bonus etc. In organizations characterized by strong knowledge sharing hostility, making mistakes has always been banned [11]. Managers do not attach enough importance to encouraging staff to considerate mistakes and give feedback, but just focus on "who is to blame", which makes employees more afraid to tell colleagues their own mistakes, which is not conducive to knowledge sharing. As a high-tech industry, the work in e- 
commerce enterprise is demanding, and management measures are also relatively harsh, which causes employees to understand mistakes biased to a certain extent, let alone share false knowledge. This leads us to hypothesize that:

H3: Passive attitudes toward false knowledge have a negative effect on knowledge sharing.

\subsection{Theory of knowledge governance}

\subsubsection{Formal knowledge governance and informal knowledge governance}

Grandori (1997) was the first person to put forward the concept of knowledge governance, he believes that knowledge governance is to use the organization system to promote intellectual activity and management knowledge exchange, transfer and sharing Inside and outside the organization [12]. Based on structure view, process view and system view, Wang (2013) studied the model and evolution process of organizational knowledge governance [13]. Li (2007) studied the dimensions of knowledge governance from the perspective of transaction cost economics [14]. From the cognitive perspective, Ren (2007) divided knowledge governance into formal and informal knowledge governance [15]. Referring to Ren's classification of knowledge governance, this paper divides knowledge governance into formal and informal knowledge governance. Ren (2007), meanwhile, pointed out that knowledge governance is that organization utilizes the formal system design to influence the informal activities within the organization so as to choose, create, share and use knowledge optimally [15]. Xiang and Cao (2014) found that formal knowledge governance has a guiding role on informal knowledge governance through the empirical research [16]. Zhao and Liu (2015), found that both formal knowledge governance and informal knowledge governance have a positive effect on the creativity of the organization through empirical research and formal knowledge governance is in a dominant position [1]. Thus:

H4: Formal knowledge governance has a positive effect on informal knowledge governance.

\subsubsection{Knowledge governance and reasons of knowledge sharing hostility}

Formal knowledge governance means that organizations use organizational design, material incentives to govern the behaviour of knowledge sharing.

Based on the theory of social exchange, employees pay more attention to the value gained in the knowledge sharing activities. So, material motivation, as a means of formal knowledge governance, can alleviate the knowledge sharing hostility among employees, and effectively promote the knowledge sharing behaviour. Jin (2011) pointed out that incentives can make employees feel that organization encourages knowledge sharing, and when they get material rewards because of knowledge sharing, their willingness of knowledge sharing will be more intense, hence material incentives have a positive effect on individual subjective intention of knowledge sharing [17]. Zhao and Liu (2015) pointed out that the intellectual rewards can not only motivate employees to share knowledge, but also stimulate them to acquire new knowledge, improve the internal staff interact with external environment actively, access to external knowledge [1]. Husted Kenneth et al. (2012) pointed out that the transaction-based mechanisms can effectively weaken employees' negative attitude towards the mistakes they have made, and organizational incentives can promote the employees to participate in the knowledge sharing actively [3]. The organization design should include the design of performance evaluation system, and involve the contribution to the efficiency of others, which helps to reduce the occurrence of knowledge hoarding behaviours [18]. Wang (2015) pointed out that the development of education and training system in the organization design, help new employees to understand the distribution of knowledge within the organization, and reduce the suspicion of other people's knowledge, to promote their participation in knowledge sharing [6]. The organization design based on team training system can weaken the negative attitude of the employees to the false knowledge, so as to promote the knowledge sharing [3].

Therefor the following hypotheses are proposed:

H5a: Formal knowledge governance has a negative effect on the reasons of knowledge hoarding.

H5b: Formal knowledge governance has a negative effect on the reasons of knowledge hoarding.

H5c: Formal knowledge governance has a negative effect on the negative attitude towards false knowledge.

H6a: The reasons of knowledge hoarding have a mediating effect between formal knowledge governance and knowledge sharing.

H6b: The reasons of knowledge rejecting have a mediating effect between formal knowledge governance and knowledge sharing.

H6c: The negative attitude towards false knowledge has a mediating effect between formal knowledge governance and knowledge sharing.

Informal knowledge governance is to use organizational culture and trust interfere knowledge sharing behaviour among employees.

Organizational culture can help employees to realize that individuals and organizations are a whole and can be integrated into the organization as soon as possible, and the culture of unity can enhance the trust among employees [19], which can diminish knowledge hoarding and knowledge rejecting and promote knowledge sharing. Organizational culture, based on trust, reduce staffs' doubts of other people's knowledge to a certain extent [6], and promote them to accept the knowledge shared by colleagues with pleasure. Wang et al. (2013) found that if employees feel that they are in a friendly, fair atmosphere in which knowledge sharing is encouraged and supported, they will be more actively 
involved in the knowledge sharing [9]. Zhang and Zhu (2012) pointed out that colleagues' attitudes towards knowledge sharing will directly affect the willingness of knowledge sharing of both sides Good interpersonal relationships among colleagues will promote knowledge sharing among employees [20]. Whether managers pay attention to the exchange of the false knowledge between employees, will directly affect the attitude to the wrong knowledge is positive [8]. Managers' creating a relaxed atmosphere of the organization will help employees to weaken the negative attitude to the mistakes they have made, and promote knowledge sharing.

On the basis of these arguments, the following hypotheses are advanced:

H7a: Informal knowledge governance has a negative effect on the reasons of knowledge hoarding.

$\mathrm{H} 7 \mathrm{~b}$ : Informal knowledge governance has a negative effect on the reasons of knowledge hoarding.

H7c: Informal knowledge governance has a negative effect on the negative attitude towards false knowledge.

H8a: The reasons of knowledge hoarding have a mediating effect between Informal knowledge governance and knowledge sharing.

$\mathrm{H} 8 \mathrm{~b}$ : The reasons of knowledge rejecting have a mediating effect between Informal knowledge governance and knowledge sharing.

H8c: The negative attitude towards false knowledge has a mediating effect between Informal knowledge governance and knowledge sharing.

According to the above assumptions, the following theoretical model is proposed. FKG stands for formal knowledge governance; IFKG represents informal knowledge governance; RKH means reasons of knowledge hoarding; RKJ denotes reasons of knowledge rejecting; AFK signifies negative attitude towards false knowledge; KS expresses knowledge sharing:

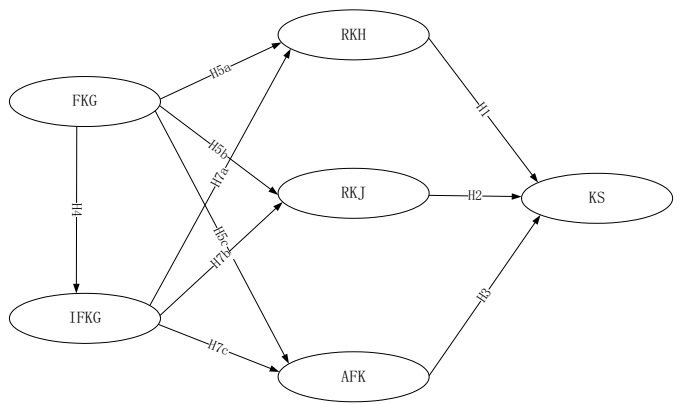

Fig. 1. Research model.

\section{Research design and hypothesis testing}

\subsection{Data source}

In this study, we use the method of questionnaire survey to collect data, and research the e-commerce enterprises in Henan province. We first conduct research for 10 individuals in each enterprise before the formal questionnaire survey, and then we amend the questionnaire according to their recommendations and research needs. The research questionnaire was sent to 200 members, with 185 respondents were returned, of which 177 questionnaires were valid. Basic statistics are shown in Table 1. EB means educational background; WL stands for work life:

Table 1. Basic statistics.

\begin{tabular}{|c|c|c|c|}
\hline \multirow{2}{*}{ title } & category & number & percentage \\
\hline \multirow{4}{*}{ age } & male & 61 & $34.5 \%$ \\
\cline { 2 - 4 } & female & 116 & $65.5 \%$ \\
\cline { 2 - 4 } & Under 25 & 80 & $45.2 \%$ \\
\hline \multirow{3}{*}{ EB } & $26-35$ & 94 & $53.1 \%$ \\
\cline { 2 - 4 } & $36-45$ & 3 & $1.7 \%$ \\
\cline { 2 - 4 } & Junior College & 65 & $36.7 \%$ \\
\hline \multirow{2}{*}{ Undergraduate } & 101 & $57.1 \%$ \\
\hline
\end{tabular}




\begin{tabular}{|c|c|c|c|}
\hline \multirow{4}{*}{ WL } & Less than a year & 88 & $49.7 \%$ \\
\cline { 2 - 4 } & $1-3$ years & 66 & $37.3 \%$ \\
\cline { 2 - 4 } & $3-6$ years & 20 & $11.3 \%$ \\
\cline { 2 - 4 } & 7 years and above & 3 & $1.7 \%$ \\
\hline
\end{tabular}

\subsection{Measurement of variables}

In this study, we measured 5 latent variables: formal knowledge governance, informal knowledge governance, reasons of knowledge hoarding, reasons of knowledge rejecting, negative attitude towards false knowledge. Measurement of knowledge governance draws Cao's (2012) measurement scale of knowledge governance and items of reasons of knowledge hoarding, reasons of knowledge rejecting, negative attitude towards false knowledge refer to Kenneth Husted et al. measurement to the knowledge sharing hostility. All items were measured by using a five-point Likert scale $(1=$ strongly disagree, $5=$ strongly agree $)$.

(1) Reliability Analysis

In this paper, we test the internal consistency of all variables by using Cronbach alpha value in reliability analysis. We used SPSS to analyse relevant data of questionnaire survey. The Cronbach 's alpha coefficient was 0.795 and the standard of the Cronbach s' alpha coefficient was 0.797 , both of which were larger than the critical value of 0.7 , which shows that the statistical results of the questionnaire have a good reliability.

(2) Validity Analysis

Validity analysis is based on the reliability analysis of the data obtained from the questionnaire to ensure the stability and consistency of the research results. The study of measurement indicators can reflect the degree of the problem characteristics to be measured. We performed the factor analysis of the data. The KMO value was 0.859 and the significant level of Bartlett ball type test is 0.000 . According to the above research, the data of this study has good reliability and validity, meeting the requirements of research.

\subsection{Empirical methods and results}

The main variables for statistical analysis and correlation analysis descriptive results are shown in Table 2. M means Mean value and SD stands for standard deviation. As can be seen from the data in Table 2, the correlation between variables is well.

Table 2. The main variables for statistical analysis and correlation analysis.

\begin{tabular}{|l|l|l|l|l|l|l|l|l|}
\hline & $\mathrm{M}$ & SD & 1 & 2 & 3 & 4 & 5 & 6 \\
\hline FKG & 18.25 & 3.453 & 1 & $0.762^{* *}$ & $-0.174^{*}$ & $-0.242^{* *}$ & $0.325^{* *}$ & $0.467^{* *}$ \\
\hline IFKG & 22.19 & 4.401 & $0.762^{* *}$ & 1 & $-0.215^{* *}$ & $-0.263^{* *}$ & $0.233^{* *}$ & $0.502^{* *}$ \\
\hline RKH & 13.31 & 3.966 & $-0.174^{*}$ & $-0.215^{*}$ & 1 & $0.679^{* *}$ & -0.003 & $-0.191^{* *}$ \\
\hline RKJ & 5.96 & 2.082 & $-0.242^{* *}$ & $-0.263^{* *}$ & $0.679^{* *}$ & 1 & 0.030 & $-0.299^{* *}$ \\
\hline AFK & 10.45 & 1.898 & $0.325^{* *}$ & $0.233^{* *}$ & -0.003 & 0.030 & 1 & $-0.251^{* *}$ \\
\hline KS & 15.06 & 2.497 & $0.467^{* *}$ & $0.502^{* *}$ & $-0.191^{*}$ & $-0.299^{* *}$ & $-0.251^{* *}$ & 1 \\
\hline
\end{tabular}

notes: $\mathrm{N}=177$; Significant level: **Express $\mathrm{P}<0.01$; $*$ Express $\mathrm{P}<0.05$

(1) The empirical study of social dilemma theory

We used the method of linear regression analysis to verify the impact of reasons for knowledge sharing hostility on knowledge sharing. The results are shown in Table 3.

Model 1 tests the relationship between reasons of knowledge hoarding and knowledge sharing. The results of analysis indicate that the reasons of knowledge hoarding have a significant, negative and weaken effect on knowledge sharing $(\beta=-0.136, \mathrm{P}=0.004)$, so $\mathrm{H} 1$ is supported by the data.

Model 2 tests the relationship between reasons of knowledge rejecting and knowledge sharing. The results of analysis indicate that the reasons of knowledge hoarding have a significant, negative and weaken effect on knowledge sharing ( $\beta=-0.401, \quad \mathrm{P}=0.000)$, so $\mathrm{H} 2$ has been verified.

Model 3 tests the relationship between negative attitude towards false knowledge and knowledge sharing. Contrary to our hypothesis, the negative attitude towards false knowledge has a positive effect on knowledge sharing ( $\beta=0.333$, $\mathrm{P}=0.001)$, so $\mathrm{H} 3$ has not been verified.

Table 3. Regression analysis of reasons of knowledge sharing hostility and knowledge sharing.

\begin{tabular}{|l|c|c|c|}
\hline \multirow{2}{*}{ variable } & \multicolumn{3}{|c|}{ KS } \\
\cline { 2 - 4 } & Model 1 & Model 2 & Model 3 \\
\hline
\end{tabular}




\begin{tabular}{|c|c|c|c|}
\hline sex & -0.526 & -0.586 & -0.388 \\
\hline age & 0.261 & 0.148 & -0.063 \\
\hline EB & -0.085 & -0.008 & -0.037 \\
\hline WL & $0.568^{*}$ & $0.632^{*}$ & $0.660^{*}$ \\
\hline OP & 0.182 & 0.230 & 0.135 \\
\hline RKH & $-0.136^{* *}$ & & \\
\hline RKJ & & $-0.401^{* *}$ & $0.333^{* *}$ \\
\hline AFK & & & 0.111 \\
\hline $\mathrm{R}^{2}$ & 0.093 & 0.155 & 0.079 \\
\hline Adjust $\mathrm{R}^{2}$ & 0.061 & 0.125 & 3.526 \\
\hline F value & 2.922 & 5.193 & \\
\hline
\end{tabular}

notes: $\mathrm{N}=177$; Significant level: **Express $\mathrm{P}<0.01 ; *$ Express $\mathrm{P}<0.05$

(2) The empirical study on knowledge governance theory

Table 4 presents the results of the regression analysis of knowledge governance theory.

Model 1 tests the relationship between formal knowledge governance and informal knowledge governance, the results show that formal knowledge governance has a significant positive effect on informal knowledge governance $(\beta=0.971, P=0.000)$. $\mathrm{H} 4$ is supported by the data.

The regression results of model 2 and model 4 suggest that the formal knowledge governance has a significant and negative effect on reasons of knowledge hoarding and rejecting $(\beta=-0.213, \mathrm{P}=0.014 ; \beta=-0.153, \mathrm{P}=0.001)$ which indicates that $\mathrm{H} 5 \mathrm{a}$ and $\mathrm{H} 5 \mathrm{~b}$ have been verified. The regression results of model 6 show that formal knowledge governance has a significant and positive effect on attitude towards false knowledge, assuming that $\mathrm{H} 5 \mathrm{c}$ has not been verified.

The regression results of model 3 and model 5 suggest that the informal knowledge governance has a significant and negative effect on reasons of knowledge hoarding and rejecting $(\beta=-0.197, \mathrm{P}=0.003 ; \beta=-0.125, \mathrm{P}=0.000)$ which indicates that $\mathrm{H} 7 \mathrm{a}$ and $\mathrm{H} 7 \mathrm{~b}$ have been verified. The regression results of model 6 show that informal knowledge governance has a significant and positive effect on attitude towards false knowledge, assuming that $\mathrm{H} 7 \mathrm{c}$ has not been verified.

Table 4. Regression analysis of theory of knowledge governance.

\begin{tabular}{|c|c|c|c|c|c|c|c|}
\hline variable & IFKG & \multicolumn{2}{|c|}{ RKH } & \multicolumn{2}{c|}{ RKJ } & \multicolumn{2}{c|}{ AFK } \\
\hline & Model 1 & Model2 & Model 3 & Model 4 & Model 5 & Model 6 & Model 7 \\
\hline sex & 0.058 & -1.191 & -1.178 & -0.559 & -0.549 & -0.479 & -0.492 \\
\hline age & 0.729 & -0.350 & -0.210 & -0.384 & -0.300 & 0.318 & 0.266 \\
\hline EB & 0.396 & -0.120 & -0.035 & 0.120 & 0.181 & 0.208 & 0.139 \\
\hline WL & -0.380 & 0.420 & 0.134 & 0.252 & 0.197 & -0.266 & -0.207 \\
\hline OP & 0.008 & $0.446^{*}$ & $0.445^{*}$ & $0.281^{*}$ & $0.278^{*}$ & 0.087 & 0.098 \\
\hline FKG & $0.971^{* *}$ & $-0.213^{*}$ & & $-0.153^{* *}$ & & $0.181^{* *}$ & \\
\hline IFKG & & & $-0.197^{* *}$ & & $-0.125^{* *}$ & & $0.096^{* *}$ \\
\hline $\mathrm{R}^{2}$ & 0.593 & 0.069 & 0.083 & 0.113 & 0.119 & 0.140 & 0.082 \\
\hline Adjust $\mathrm{R}^{2}$ & 0.579 & 0.037 & 0.051 & 0.082 & 0.088 & 0.110 & 0.050 \\
\hline F value & 41.311 & 2.116 & 2.564 & 3.623 & 3.830 & 4.625 & 2.546 \\
\hline
\end{tabular}

notes: $\mathrm{N}=177$; Significant level: **Express $\mathrm{P}<0.01$; $*$ Express $\mathrm{P}<0.05$

(3) The empirical study on the mediating effect of reasons of knowledge sharing hostility

Table 5 shows the mediating effect of reasons of knowledge sharing hostility.

the results of table 4 show that formal knowledge governance has a significant positive effect on informal knowledge governance; formal and informal knowledge governance have a significant impact on the reasons of knowledge hoarding and rejecting and negative attitude towards false knowledge; the test of mediating effect table 5 model 3 and model 4, while adding formal and informal knowledge governance (independent variables) and reasons of knowledge hoarding, reasons of knowledge rejecting and negative attitude towards false knowledge (mediator) to predict knowledge sharing (dependent variable). Reasons of knowledge rejecting and negative attitude towards false knowledge have a significant effect knowledge sharing. Moreover, the effect of formal and informal knowledge governance is 
significant and coefficients are reduced, which indicates that the reasons of knowledge rejecting and passive attitudes towards failure knowledge exist partial mediating effect between formal and informal knowledge government and knowledge sharing. On the contrary, the reasons of rejecting have no mediating effect between formal and informal knowledge governance and knowledge sharing.

Table 5. Regression analysis the mediating effect of reasons of knowledge sharing hostility.

\begin{tabular}{|c|c|c|c|c|}
\hline \multirow{2}{*}{ variable } & \multicolumn{2}{|c|}{ KS } & \multicolumn{2}{c|}{ KS } \\
\cline { 2 - 5 } & Model 1 & Model 2 & Model 3 & Model 4 \\
\hline sex & -0.339 & -0.36 & -0.406 & -0.386 \\
\hline age & 0.247 & 0.053 & 0.070 & -0.094 \\
\hline EB & 0.041 & -0.09 & 0.042 & -0.063 \\
\hline WL & 0.463 & 0.583 & $0.590^{*}$ & $0.687^{* *}$ \\
\hline OP & 0.082 & 0.087 & 0.148 & 0.138 \\
\hline FKG & $0.327^{* *}$ & & $0.248^{* *}$ & \\
\hline IFKG & & $0.281^{* *}$ & & $0.227^{* *}$ \\
\hline RKH & & & 0.018 & 0.032 \\
\hline RKJ & & & $-0.319^{* *}$ & $-0.315^{* *}$ \\
\hline AFK & & & $0.189^{*}$ & $0.221^{*}$ \\
\hline $\mathrm{R}^{2}$ & 0.251 & 0.291 & 0.361 & 0.358 \\
\hline Adjust $\mathrm{R}^{2}$ & 0.224 & 0.266 & 0.279 & 0.324 \\
\hline F value & 9.477 & 11.647 & 8.558 & 10.366 \\
\hline
\end{tabular}

notes: $\mathrm{N}=177$; Significant level: **Express $\mathrm{P}<0.01$; *Express $\mathrm{P}<0.05$

\section{Discussions and limitations}

\subsection{Discussions}

This paper discusses governance mechanism of knowledge sharing hostility in the e-commerce enterprise. The research results are as follows:

(1) The effect of reasons of knowledge sharing hostility on knowledge sharing

In e-commerce enterprises, the analysis results in Table 3 show that the reasons of knowledge hoarding and rejecting have a significant and negative effect on knowledge sharing, but negative attitude towards false knowledge has a significant and positive effect on knowledge sharing. When analysing three factors' influence on knowledge sharing, we found that the working life has a significant positive effect on knowledge sharing $(\beta=0.568, \mathrm{P}=0.031 ; \beta=0.632, \mathrm{P}=0.013 ; \beta=0.660, \mathrm{P}=0.018)$. Thus, those employees who are experienced and senior are more willing to share knowledge. Therefore, during the knowledge governance, organization can establish an interdependent system to enable older employees to help new employees, and to find the reasons for knowledge sharing hostility among new employees so as to promote knowledge sharing through psychological counselling for them. In addition, the three model's goodness of fittest is not $\operatorname{good}(\mathrm{R} 2=0.093 ; \mathrm{R} 2=0.155 ; \mathrm{R} 2=0.111)$. One possible explanation is that there exist other factors in knowledge sharing, such as relationships among employees within organization etc.

(2) The effect of formal and informal knowledge governance on reasons knowledge sharing hostility

Through the analysis of the data, it verifies that formal knowledge governance has a significant positive effect on informal knowledge governance; formal and informal knowledge governance have a significant and passive impact on reasons of knowledge hoarding and rejecting. Contrary to our hypothesis, the effect of knowledge governance on the negative attitude towards the false knowledge is positive. This may be because the company's managers pursue the perfect and corporation's governance mechanism is too harsh, so employees fear to be punished because of making mistakes. Therefore, managers should fully consider the feelings of employees when making knowledge governance system, and they should also encourage employees to share the experience learning from the mistakes. In our study, we found that operating post has a significant positive effect on reasons of knowledge hoarding and knowledge rejecting from the analysis in Table 4. One possible explanation of the reasons may be that knowledge fields or background span is relatively large among different jobs, and employees may be not familiar with each other, which promote the behaviour of knowledge sharing hostility. Huang (2009) pointed out that the trust mechanism can enhance transactive memory and promote knowledge sharing. Therefore, organization can establish transactive memory system in 
knowledge governance mechanism in which employees can strengthen the exchange of knowledge and know more about what kind of knowledge colleagues grasp [21]. At work, if you do not know a piece of knowledge, you can ask your colleagues who are master of this filed to solve problems.

(3) The mediating effect of reasons of knowledge sharing hostility

Through the analysis of the data, the reasons of knowledge rejecting and the negative attitude towards the false knowledge have partial mediating effect between formal and informal knowledge governance and knowledge sharing. However, R2 value of 0.361 indicates that the goodness of fitness is not well. The possible reason is the limited number of our questionnaire, and the sample size is not big enough, so this result may be change after increasing sample size, and reasons of knowledge sharing hostility may exist partial or total mediating effect between formal and informal knowledge governance and knowledge sharing.

\subsection{Limitations}

(1) We didn't distinguish the same department or cross functional departments when studying the governance mechanism's impact on knowledge sharing hostility, only taking into account behaviours knowledge sharing in hostile in the same organization. While knowledge sharing is a dynamic process, the causes of the knowledge sharing between the same department and the cross sector may be different, and the further studies can be carried out in this area.

(2) The thought of Internet + and The Belt and Road make the development environment of e-commerce enterprises more uncertain. In this paper, we do not take into account the internal external environment for the organization, but any governance of an enterprise cannot be separated from the environment of the organization.

(3) In the research of knowledge sharing behaviour, social capital is also a more important point. In this paper, we didn't consider social capital, so the further research should examine the effect of social capital on reasons of knowledge sharing hostility.

\section{Acknowledgement}

This research was conducted with the support of key scientific research projects of institution of higher learning in Henan Province in 2016.

\section{References}

1. Zhao, X. and Liu, X.M., The promoting mechanism of social knowledge governance on organizational creativity, Science of Science and Management of S. \& T., Vol. 36 No. 6, pp. 94-97, 2015.

2. Li,J., Modern Finance \& Economics, Research on the governance mechanism of knowledge sharing in large scale collaboration, Vol. 30 No. 9, pp.69-73,(2010)

3. Kenneth H., Snejina M., Dana B.M. and Torben P., Knowledge-sharing hostility and governance mechanisms: an empirical test, Journal of Knowledge Management, Vol. 10 No. 5, pp.754-773, 2012.

4. Luo, M., Zhang, S.Y. and Liu, Y.J., The research on new and high technology enterprise's knowledge governance performance,Science Research Management, Vol. 31 No. 3, pp.1-9, 2010.

5. Heng-Li Yang, Ted C.T. Wu. Knowledge sharing in an organization, Technological Forecasting \& Social Change, No.75, pp.1128-1156,2008.

6. Wang, R.H. and Lv, Y.B., Research on the governance mechanisms of mentoring knowledge sharing hostility, Research on Library Science, No.21, pp. 96-101, 2015.

7. Chen, T., Wang, T.N. and Zhu, Z.M., The distance of knowledge, environmental turbulence and the relationship of knowledge sharing in organization: an empirical research which contains moderating effect, Studies in Science of Science, Vol. 31 No. 10, pp. 1532-1539,2013.

8. Snejina, M., Kenneth, H., Knowledge-Sharing Hostility in Russian Firms, California Management Review, Vol. 45 No.3, pp. 59-75, 2003.

9. Wang, S.H., Xu, B. and Peng, J.S., The impact of organizational climate perception on employees innovation behavior: Based on the medium role of knowledge sharing intention, Science Research Management, Vol.34 No.5, pp. 130-135, 2013.

10. Diaz-Diaz, N., Aguiar-Diaz, I. and De Saa-Perez, P., the effect of technological knowledge assets on performance: the innovation in Spanish firms, Research Policy, Vol.37 No.9, pp. 1515-1529, 2008.

11. Husted, K. and Michailova, S., Diagnosing and fighting knowledge sharing hostility, Organizational Dynamics, Vol.31 No.1, pp. 60-73,2002.

12. Grandori, A.,Governance structures, coordination mechanisms and cognitive models, Journal of Management and Governance, Vol.1 No.1, pp. 29-42, 1997. 
13. Wang, Y., Research on knowledge governance pattern of organization and its evolutionary process, Forum on Science and Technology in China, No.3, pp. 102-108, 2013.

14. Li, Y., New development of governance: latitude analysis of knowledge management, Jiangxi Social Science, pp. 173-176,2007.

15. Ren, Z.A., Go beyond knowledge management: Conceptions, framework and application of knowledge governance theory, Science Research Management, Vol.28 No.1, pp. 20-26,2007.

16. Cao, Y. and Xiang, Y., Study on the relationship among knowledge governance, knowledge sharing and employee innovation based on the mediating of social capital and the moderating of absorptive capacity in enterprises, Studies in Science of Science, Vol.32 No.1, pp. 92-102, 2014.

17. Jin, H., Yang, Z. and Feng, F., Rewards, perceived ownership of knowledge and organizational knowledge sharing incentive mechanism, Studies in Science of Science, Vol.29 No.7, pp. 1036-1055, 2011.

18. Zhang, C.Z., Jiang, X.R. and Xu, H.B., Research on the influence of organizational design on knowledge sharing, Science \& Technology Progress and Policy, Vol.30 No.3, pp. 128-133, 2013.

19. Jin, H., Yang, Z., Huang, Y.T. and Wu, J., Exploring how organizational incentives and organizational culture affect knowledge sharing based on modified SIT, Studies in Science of Science, Vol.31 No.11, pp. 1697-1707, 2013.

20. Zhang, X.D. and Zhu, M., The impact of incentive, attitude toward colleagues, and personal culture on the knowledge sharing, Science Research Management, Vol.33 No.10, pp. 97-105, 2012.

21. Chi-Cheng Huang. Knowledge sharing and group cohesiveness on performance: An empirical study of technology $R \& D$ teams in Taiwan, Technovation, No.29, pp.786-797, 2009. 